\title{
New species of the millipede genus Glypbiulus Gervais, 1847 from the javanicus-group (Diplopoda: Spirostreptida: Cambalopsidae)
}

\author{
Новые виды диплопод рода Glypbiulus Gervais, 1847 из группы \\ javanicus (Diplopoda: Spirostreptida: Cambalopsidae)
}

\section{S.I. Golovatch ${ }^{1}$, J.-J. Geoffroy ${ }^{2}$, J.-P. Mauriès ${ }^{3} \&$ D. VandenSpiegel ${ }^{4}$ С.И. Головач ${ }^{1}$ ЖК.-Ж. ЖКоффруа ${ }^{2}$ ЖК.-П. Морьес ${ }^{3}$, А. ВанденШпигель ${ }^{4}$}

\author{
${ }^{1}$ Institute for Problems of Ecology and Evolution, Russian Academy of Sciences, Leninsky pr. 33, Moscow 119071, Russia. \\ ${ }^{1}$ Институт проблем экологии и эволюции РАН, Ленинский пр., 33, Москва 119071 Россия. \\ ${ }^{2}$ Muséum national d'Histoire naturelle, Département Ecologie \& Gestion de la Biodiversité, UMR 7204 CERSP du CNRS, Equipe \\ EVOLTRAIT, 4, avenue du Petit Château, F-91800 Brunoy, France. \\ ${ }^{3}$ Muséum national d'Histoire naturelle, Département Systématique et Evolution, Section Arthropodes, Case Postale n53, 61 rue Buffon, \\ F-75231 Paris Cedex 05, France. \\ ${ }^{4}$ Musée Royal de l'Afrique centrale, B-3080 Tervuren, Belgium.
}

KEY WORDS: Diplopoda, Glyphiulus, taxonomy, new species, cave, Vietnam, China.

КЛЮЧЕВЫЕ СЛОВА: Diplopoda, Glyphiulus, таксономия, новый вид, пещера, Вьетнам, Китай.

\begin{abstract}
The javanicus-group of the basically southeast Asian genus Glyphiulus is shown to currently comprise 22 species, of which four are new: G. echinoides sp.n., from a cave in Guangxi Province, China, as well as G. subechinoides sp.n., G. parechinoides sp.n. and G. submediator sp.n., all three latter species from Vietnam. Besides this, a new record is provided, and additional abundant illustrations are presented, for G. mediobliteratus Golovatch, Geoffroy, Mauriès \& VandenSpiegel, 2007, from a cave in northeastern Vietnam. The species G. vietnamicus Mauriès, 1977, from a cave in northern Vietnam, is formally ejected from the javanicus-group, instead being transferred to $\mathrm{Hy}$ pocambala Silvestri, 1897; this results in $H$. vietnamica (Mauriès, 1977), comb.n.
\end{abstract}

РЕЗЮМЕ. Группа видов javanicus исконно юговосточноазиатского рода Glyphiulus теперь содержит 22 вида, включая четыре новые: G. echinoides sp.n. из пещеры в провинции Гуанси (южный Китай), G. subechinoides sp.n., G. parechinoides sp.n. и G. submediator sp.n., все три последних вида из Вьетнама. Кроме того, зарегистрирована и богато проиллюстрирована новая находка G. mediobliteratus Golovatch, Geoffroy, Mauriès \& VandenSpiegel, 2007 в пещере на северо-востоке Вьетнама. Вид G. vietnamicus Maurius, 1977 из пещеры в Северном Вьетнаме формально исключен из группы javanicus и переведен в состав рода Hypocambala Silvestri, 1897, в результате чего возникает $H$. vietnamica (Mauriès, 1977), comb.n.

\section{Introduction}

The millipede genus Glyphiulus Gervais, 1847 has recently been redefined, reviewed and shown to comprise 39 species in two groups, the granulatus- and the javanicus-group [Golovatch et al., 2007a, b]. The granulatus-group alone has since been considerably enriched to currently encompass 33 species ranging from southern China, northern Laos and northern Thailand in the north to southern Vietnam in the south [Golovatch et al., 2011b]. Both species groups are parapatric, their ranges vastly overlapping across much of southern China and peninsular Indochina [Golovatch et al., 2007a, b, 2011b]. The isolated occurrence of Glyphiulus javanicus Carl, 1911 in eastern Java, Indonesia, where it was found in a sugar cane plantation, is probably the result of an introduction from a more northerly location, somewhere in southeast Asia.

The javanicus-group of Glyphiulus is characterized by the following features [Golovatch et al., 2007b]:

1. $\sigma^{7}$ legs 1 are nearly normal to reduced, never hypertrophied; telopodites often complete or nearly so, even when reduced in size, usually multisegmented; coxae or coxosternum with a pair of small but evident, central, medially nearly always contiguous, sometimes partly even fused outgrowths or processes which are never coalesced completely into a strong and long sickle-shaped structure (NB: a few species known only from female material are being assigned here only provisionally). 
2. $\sigma^{7}$ legs 2 virtually normal, not enlarged.

3. Typical carinotaxy patterns of the collum are I$\mathrm{III}(\mathrm{IV}, \mathrm{V})+\mathrm{P}+\mathrm{M}+\mathrm{P}+(\mathrm{V}, \mathrm{IV}) \mathrm{III}-\mathrm{I}$ or, as in the granulatus-group, I-VI+7a+pc+ma+pc+7a+VI-I, or something close. The patterns are often different, often due to crest reduction (especially anteriorly), but a median crest is always visible at least near the caudal margin.

4. The typical carinotaxy pattern of the metaterga is $2(1) / 2(1)+I / i+3 / 3+I / i+2(1)$, thus reflecting the fact that all or most of the crests are usually divided transversely into two halves. In contrast to species of the granulatus-group, the median crest is never doubled anteriorly, but sometimes it is more or less strongly reduced. If the pattern is different, it can usually be readily derived from the typical one (or vice versa), i.e. the lateral crests are reduced to one or the crests are undivided (see also below), etc.

5. The anterior gonopods are usually reduced to a plate-like coxosternum with moveable, lateral, 1-segmented, often curved telopodites (shared with some other species groups).

6 . The posterior gonopods are usually strongly compressed, often showing a subflagelliform, sometimes plumose, distal flagellum (shared with some other species groups).

7. The pleural flaps behind the gonopod opening on male segment 7 usually form a clear, transverse, ventral ridge (shared with some other species groups).

In a recent review of the genus Plusioglyphiulus Silvestri, 1923, Golovatch et al. [2011a] revealed two species from Thailand which are transitional to the javanicus-group. This genus differs now from the javanicus-group only in the presence of a clear-cut fovea on each posterior gonopod, a shaky character that might not hold given certainly numerous further members of the latter group still to be found and described.

The javanicus-group also shows certain similarities to the long accepted genus Hypocambala Silvestri, 1897, which currently contains ten species ranging from the Seychelles, Comoro Islands and Mauritius in the Indian Ocean, through Indochina and the Indo-Australian Archipelago, to New Caledonia, Loyalty Islands, Tonga and Samoa in the Pacific [Jeekel, 1963, 2004]. Indeed, the sole distinction between Glyphiulus s.1. and Hypocambala species lies in the lack in the latter group of tergal crests, tergal pilosity being present instead.

When describing G. vietnamicus, Mauriès [1977] chose to place it into Glyphiulus, rather than Hypocambala (which is represented in Vietnam by $H$. gracilis (Attems, 1938)), only because the collum and following metaterga in G. vietnamicus were carinate. However, based on the structure of $\sigma^{\gamma}$ legs 1 alone, which show virtually contiguous coxae supplied with evident distomedian outgrowths and complete telopodites, $G$. vietnamicus is clearly a species of Hypocambala, whereas the carinotaxy patterns of the collum and following metazona are definitely not generic, but only specieslevel characters [Golovatch et al., 2011c]. In other words, we eject $G$. vietnamicus from the javanicus-group [cf.
Golovatch et al., 2007b] to formally transfer it to Hypocambala, comb.n.

The present paper puts on record another four new species from the javanicus-group, but, since none of these shows a fovea on the posterior gonopod, the status of Plusioglyphiulus remains unchanged.

Abbreviations used:

IZAS - Institute of Zoology, Academia Sinica, Beijing, China;

MNHN - Muséum national d'Histoire naturelle, Paris, France;

NMNHS - National Museum of Natural History, Sofia, Bulgaria;

ZMUC - Natural History Museum, University of Copenhagen, Copenhagen, Denmark;

ZMUM - Zoological Museum, State University of Moscow, Moscow, Russia;

SCAU - South China Agricultural University, Guangzhou, China;

SEM - Scanning electron microscopy.

\section{Material and methods}

The material serving as the basis for the present contribution derives from the predominantly subterranean collections made in Vietnam and China by Anne Bedos and Louis Deharveng (MNHN), and in Vietnam by Petar Beron and Dimitar Kojuharov (NMNHS). This material has been shared between the collections of MNHN, NMNHS, IZAS, SCAU, ZMUM and ZMUC, as indicated hereafter.

SEM micrographs were taken using a JEOL JSM6480LV scanning electron microscope. After examination, SEM material was removed from stubs and returned to alcohol, all such samples being kept at MNHN.

Carinotaxy formulas are basically those developed by Golovatch et al. [2007a, b]. In addition, the numerous illustrations aid immensely in properly interpreting the formulas and in comparing the species.

\section{Taxonomic part}

\section{Glyphiulus echinoides sp.n.}

Figs 1-4.

MATERIAL. Holotype $\sigma^{\top}$ (IZAS), China, Guangxi Prov., Fushui County, Bapen Village, Cave 2, hand collection, 6.03.2005, leg. L. Deharveng \& A. Bedos (CHIgx05-045). Paratypes: 2 fragm., 1 juv. (SCAU), 1 ऽ fragm. (MNHN GA 088), 1 \% fragm. (ZMUM), $1 \sigma^{T}$ (SEM), same locality, together with holotype.

NAME. To emphasize the spiny appearance due to sharp tubercles on metazona.

DIAGNOSIS. Distinguished by the presence of only complete carinae on the collum, combined with unusually sharp tubercles on metazona, the rather polytrichous gnathochilarium, the peculiar shapes of the epiproct, paraprocts, anterior gonopods etc. Keys out as G. pulcher (Loksa, 1960), also from a cave in Guangxi Province, but differs in the anterior gonopod 

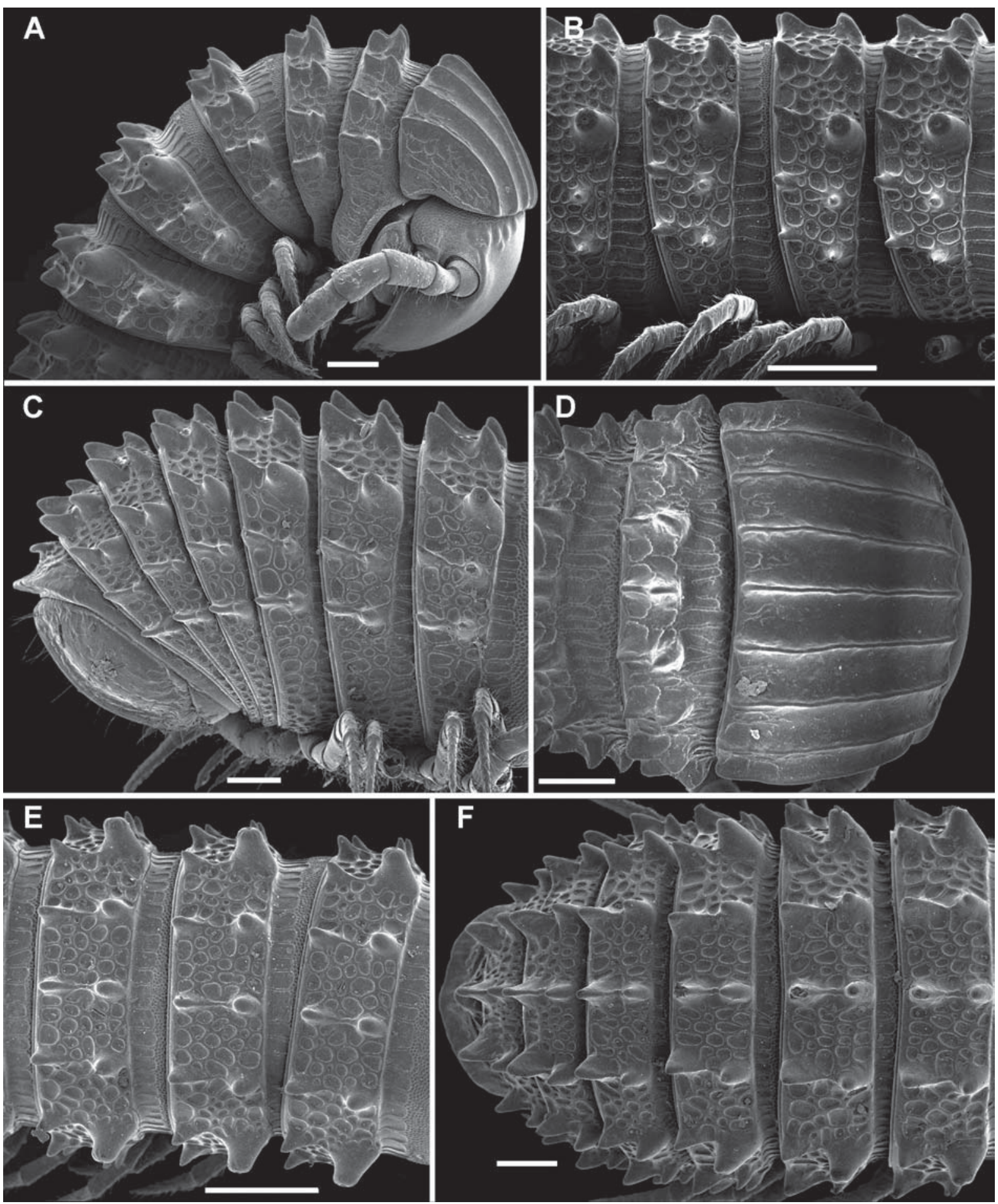

Fig. 1. Glyphiulus echinoides sp.n., $0^{7}$ paratype: A \& D - anterior part of body, lateral and dorsal views, respectively; B \& E midbody segments, lateral and dorsal views, respectively; C \& F - posterior part of body, lateral and dorsal views, respectively. Scale bars: A, C, D \& F $-0.2 \mathrm{~mm}$; B \& E $-0.5 \mathrm{~mm}$

Рис. 1. Glyphiulus echinoides sp.n., паратип О: А, D - передняя часть тела, соответственно сбоку и сверху; В, Е среднетуловищные сегменты, соответственно сбоку и сверху; C, F - задняя часть тела, соответственно сбоку и сверху. Масштаб: A, C, D, F-0,2 мм; B, E-0,5 мм. 

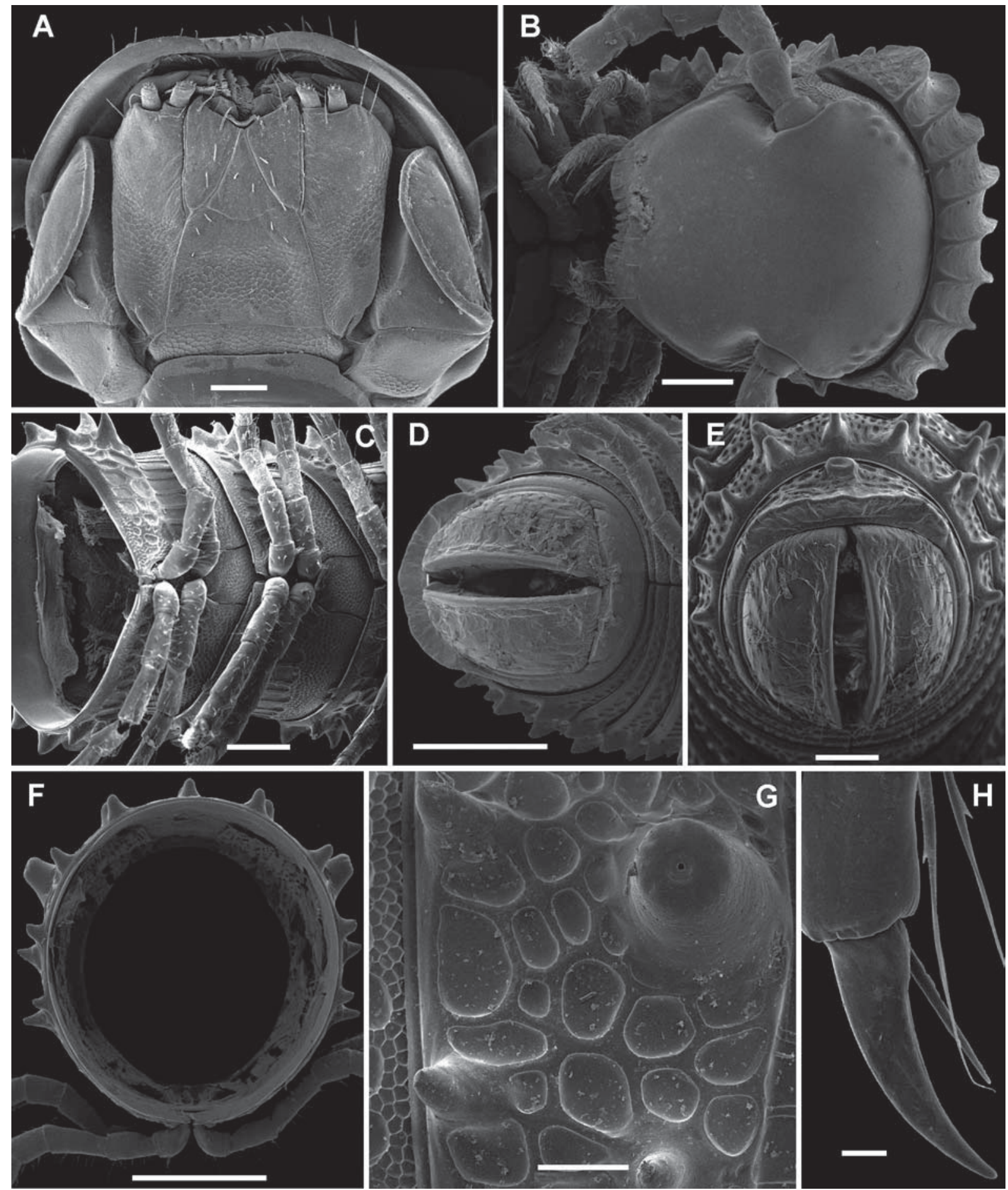

Fig. 2. Glyphiulus echinoides sp.n., $\sigma^{7}$ paratype: A — gnathochilarium, ventral view; B — head, front view; C — midbody segments, ventral view; D \& E - telson, ventral and caudal views, respectively; F - cross-section of a midbody segment, caudal view; $\mathrm{G}$ - enlarged ozopore region, lateral view; $\mathrm{H}$ - enlarged claw, lateral view. Scale bars: A, G - $0.1 \mathrm{~mm} ; \mathrm{B}, \mathrm{C}, \mathrm{E}-0.2 \mathrm{~mm}$; D, F - $0.5 \mathrm{~mm}$; H - 0.01 $\mathrm{mm}$.

Рис. 2. Glyphiulus echinoides sp.n., паратип О': А - гнатохилярий, снизу; В - голова, спереди; С - среднетуловищные сегменты, снизу; D, E - тельсон, соответственно снизу и сзади; F - поперечный разрез через среднетуловищный сегмент, сзади; $\mathrm{G}$ - увеличенный район озопоры, сбоку; $\mathrm{H}$ - увеличенный коготок, сбоку. Масштаб: A, G - 0,1 мм; B, C, E - 0,2 мм; D, F - 0,5 мм; $\mathrm{H}-0,01$ мм. 

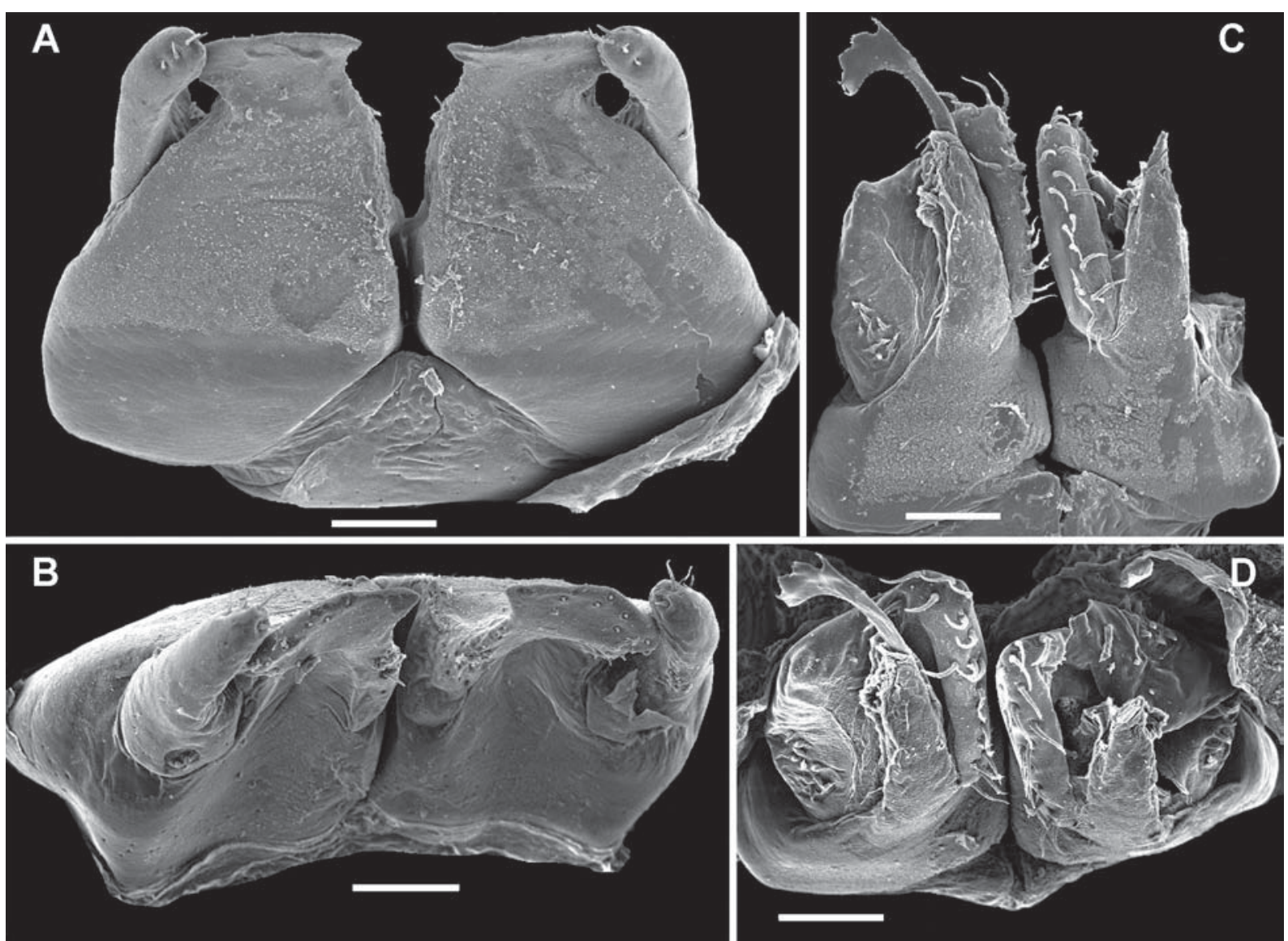

Fig. 3. Glyphiulus echinoides sp.n., O paratype: A \& B — anterior gonopods, front and ventrocaudal views, respectively; C \& D posterior gonopods, front and anteroventral views, respectively. Scale bars: $0.05 \mathrm{~mm}$.

Pис. 3. Glyphiulus echinoides sp.n., паратип О': А, В - передние гоноподы, соответственно спереди, а также снизу и сзади; С, $\mathrm{D}$ - задние гоноподы, соответственно спереди, а также спереди и снизу. Масштаб: 0,05 мм.

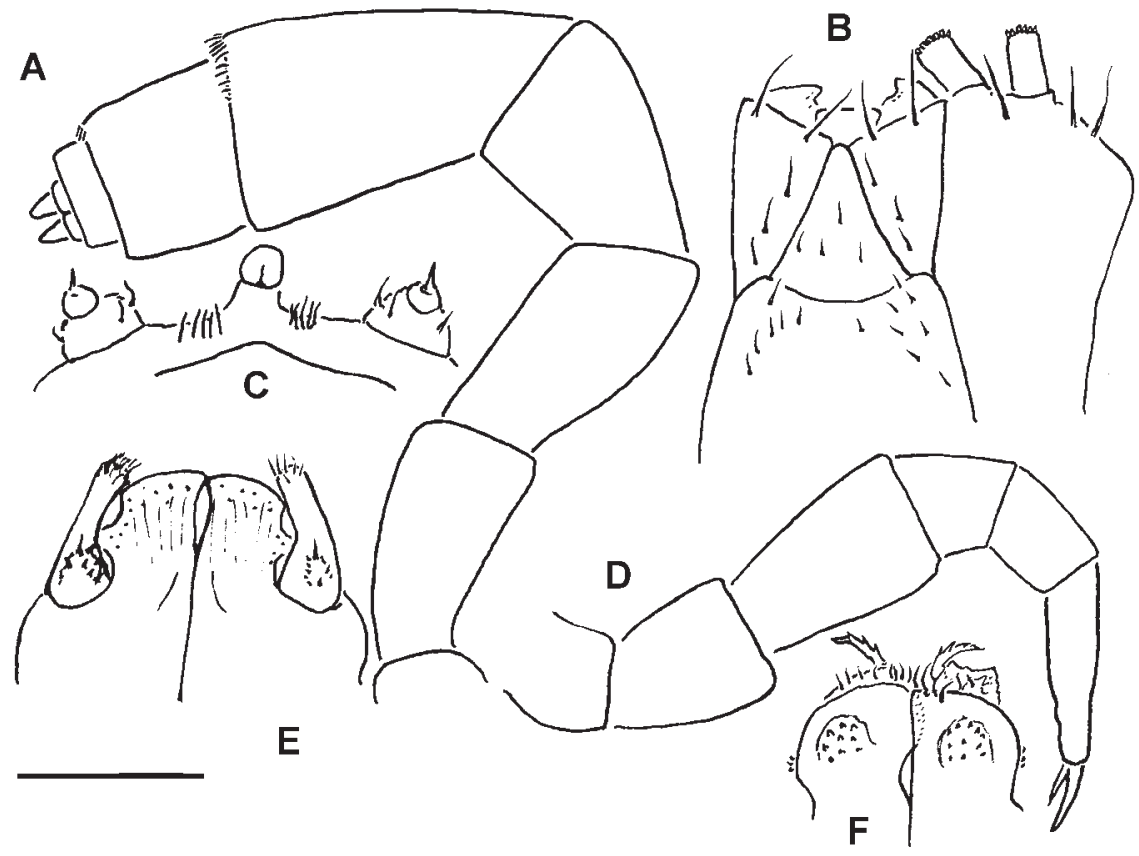

Fig. 4. Glyphiulus echinoides sp.n., O' paratype: A antenna, lateral view; $\mathrm{B}-$ gnathochilarium, ventral view; $\mathrm{C}-$ legs 1 , front view; D leg 2, caudal view; E - anterior gonopods, caudal view; F posterior gonopods, caudal view. Scale bar: $0.2 \mathrm{~mm}$.

Рис. 4. Glyphiulus echinoides sp.n., паратип $\mathrm{O}^{7}$ : A антенна, сбоку; В - гнатохилярий, снизу; $\mathrm{C}-$ ноги 1 , спереди; D - нога 2, сбоку, E - передние гоноподы, сзади; F - задние гоноподы, сзади. Масштаб: 0,2 мм. 

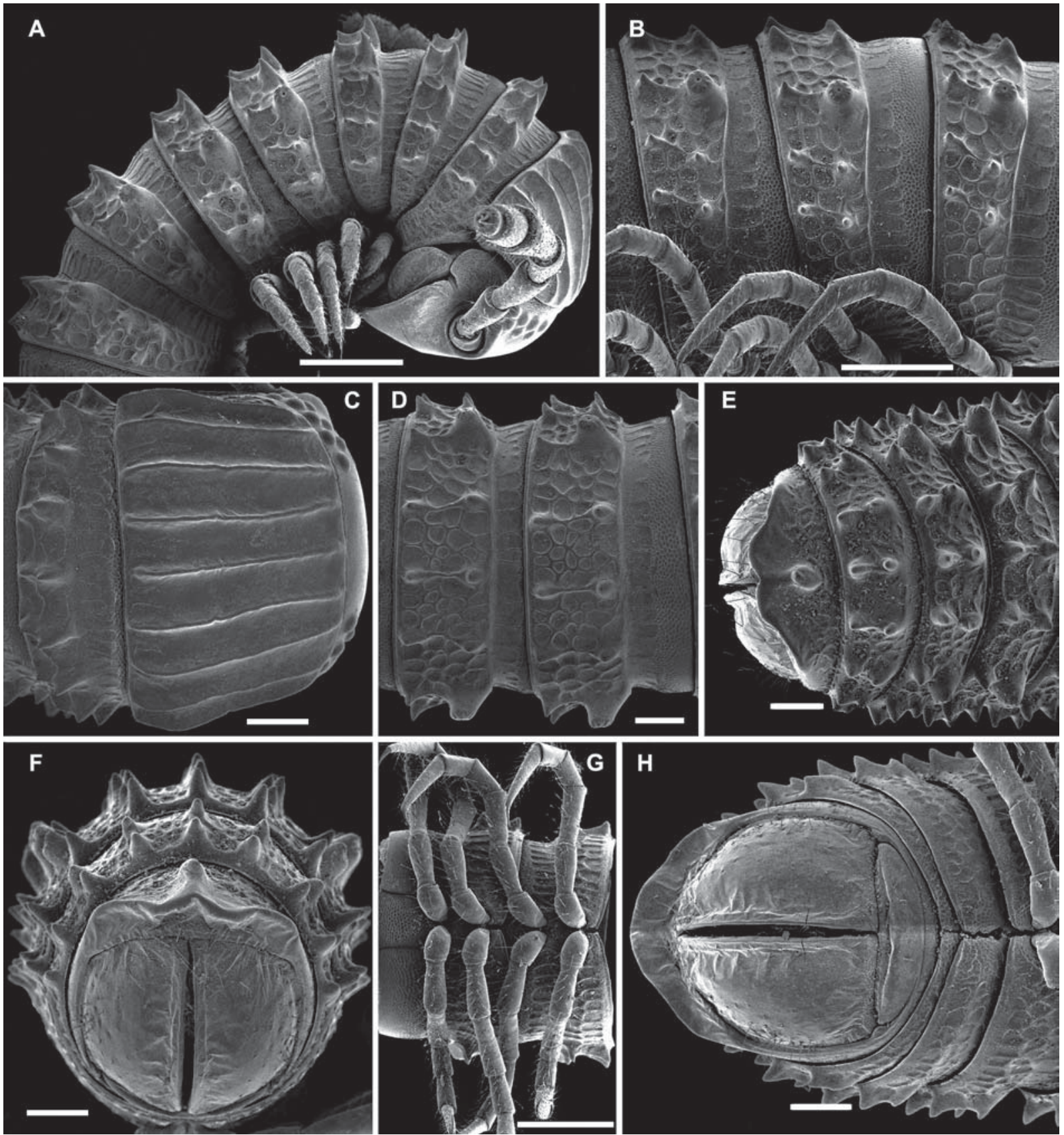

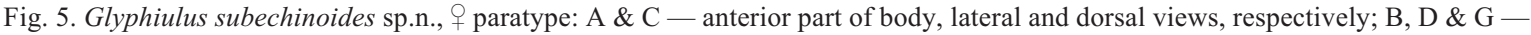
midbody segments, lateral, dorsal and ventral views, respectively; E, F \& H - posterior part of body, dorsal, caudal and ventral views, respectively. Scale bars: A, B, G-0.5 mm; C-F, H-0.2 mm.

Рис. 5. Glyphiulus subechinoides sp.n., паратип + : А, С - передняя часть тела, соответственно сбоку и сверху; В, D, G среднетуловищные сегменты, соответственно сбоку, сверху и снизу; Е, F, Н - задняя часть тела, соответственно сверху, сзади и снизу. Масштаб: A, B, G-0,5 мм; C-F, H-0,2 мм.

coxosternum being higher than the telopodites [Loksa, 1960].

DESCRIPTION. Length of holotype (the only complete specimen in the series) ca $33 \mathrm{~mm}$, width $1.5 \mathrm{~mm}$, with $58 \mathrm{p}+4 \mathrm{a}+\mathrm{T}$, midbody segments being broadest. Largest + paratype $2.0 \mathrm{~mm}$ wide. Coloration uniformly brown to dark brown.
Blackish ocelli (7?9) arranged in 1-2 irregular vertical row. Antennae short and moderately clavate (Figs 1A, 4A), extending beyond segment 3 laterally, antennomeres 6 and 7 each with a small distodorsal field of bacilliform sensilla. Gnathochilarium with a separate promentum, rather polytrichous (Figs 2A, 4B). 

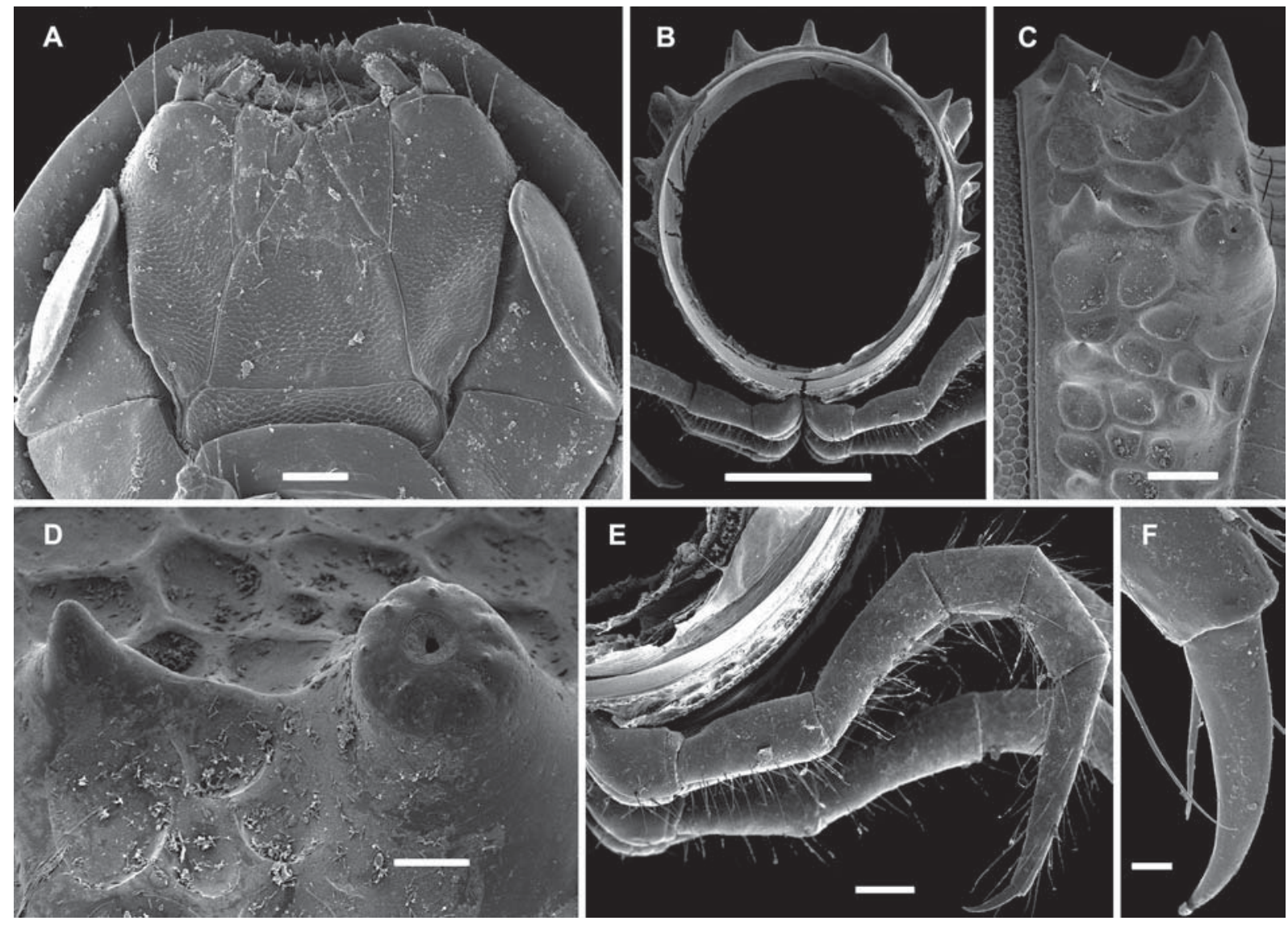

Fig. 6. Glyphiulus subechinoides sp. $\mathrm{n}$, O paratype: A — gnathochilarium, ventral view; B — cross-section of a midbody segment, caudal view; C \& D — ozopore region, lateral views; E — midbody legs, caudal view; F — enlarged claw, lateral view. Scale bars: A, C, E $0.1 \mathrm{~mm} ; \mathrm{B}-0.5 \mathrm{~mm} ; \mathrm{D}-0.05 \mathrm{~mm} ; \mathrm{F}-0.01 \mathrm{~mm}$.

Рис. 6. Glyphiulus subechinoides sp.n., паратип +: А — гнатохилярий, снизу; В — поперечный разрез через среднетуловищный сегмент, сзади; C, D - район озопоры, сбоку; Е — среднетуловищные ноги, сзади; F - увеличенный коготок, сбоку. Масштаб: А, $\mathrm{C}, \mathrm{E}-0,1$ мм; $\mathrm{B}-0,5$ мм; D - 0,05 мм; F - 0,01 мм.

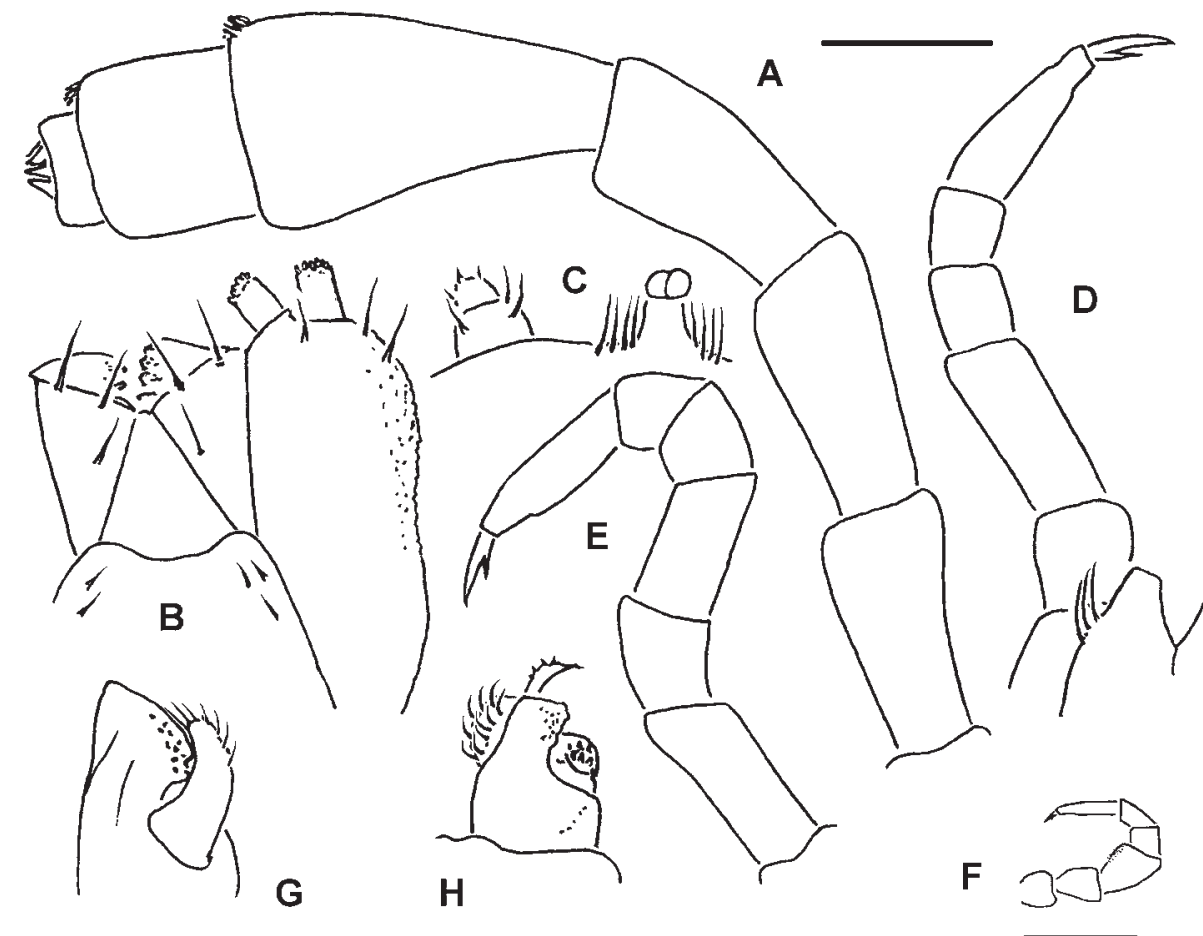

Fig. 7. Glyphiulus subechinoides sp.n., O paratype: A antenna, lateral view; $\mathrm{B}$ - gnathochilarium, ventral view; C legs 1, front view; D - leg 2, caudal view; E - leg 3, caudal view; F - leg 7, caudal view; $\mathrm{G}$ - right anterior gonopod, caudal view; $\mathrm{H}$ - left posterior gonopod, front view. Scale bars: AE, G, $\mathrm{H}-0.2 \mathrm{~mm} ; \mathrm{F}-0.5 \mathrm{~mm}$.

Рис. 7. Glyphiulus subechinoides sp.n., паратип $\mathrm{O}^{7}$ : А антенна, сбоку; В - гнатохилярий, снизу; С - ноги 1, спереди; D - нога 2, сзади; Е нога 3, сзади; F - нога 7, сзади; $\mathrm{G}$ - правый передний гонопод, сзади; $\mathrm{H}$ - левый задний гонопод, спереди. Масштаб: A-E, G, H-0,2 мм; F 0,5 мм. 

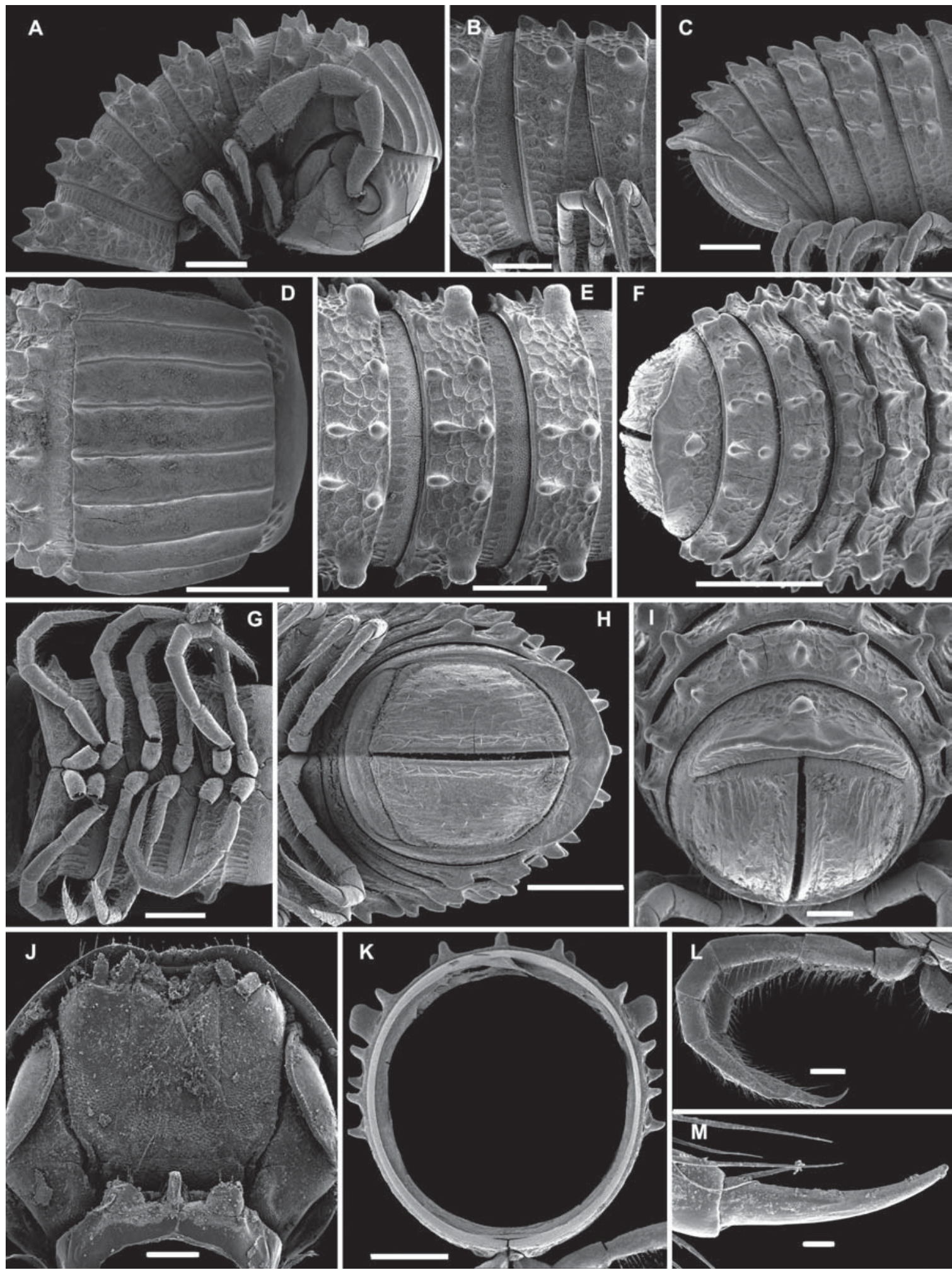

Fig. 8. Glyphiulus parechinoides sp.n., O paratype: A \& D - anterior part of body, lateral and dorsal views, respectively; B, E \& G midbody segments, lateral, dorsal and ventral views, respectively; C, F, H \& I - posterior part of body, lateral, dorsal, ventral and caudal views, respectively; $\mathrm{J}$ - gnathochilarium and legs 1 , ventral view; $\mathrm{K}$ - cross-section of a midbody segment, caudal view; $\mathrm{L}$ - midbody legs, caudal view; M - enlarged claw, lateral view. Scale bars: A-H \& K, $0.5 \mathrm{~mm}$; I, J \& L, $0.2 \mathrm{~mm}$; M, $0.02 \mathrm{~mm}$.

Рис. 8. Glyphiulus parechinoides sp.n., паратип О7: А, D - передняя часть тела, соответственно сбоку и сверху; В, E, G среднетуловищные сегменты, соответственно сбоку, сверху и снизу; С, F, Н, I - задняя часть тела, соответственно сбоку, сверху, снизу и сзади; J - гнатохилярий и ноги 1 , снизу; $\mathrm{K}$ - поперечный разрез через среднетуловищный сегмент, сзади; $\mathrm{L}-$ среднетуловищные ноги, сзади; М — увеличенный коготок, сбоку. Масштаб: А-H, K - 0,5 мм; I, J, L — 0,2 мм; M - 0,02 мм. 

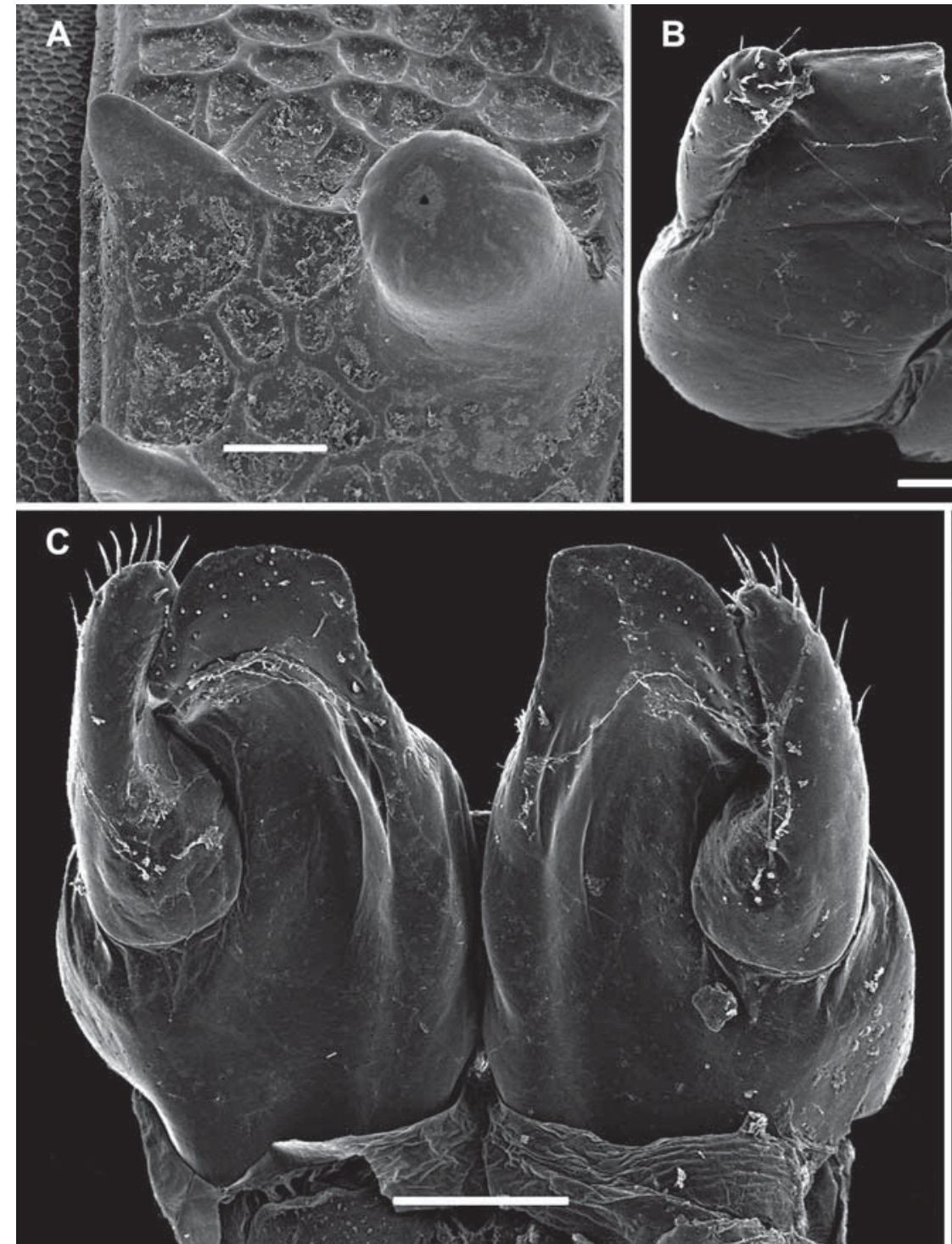
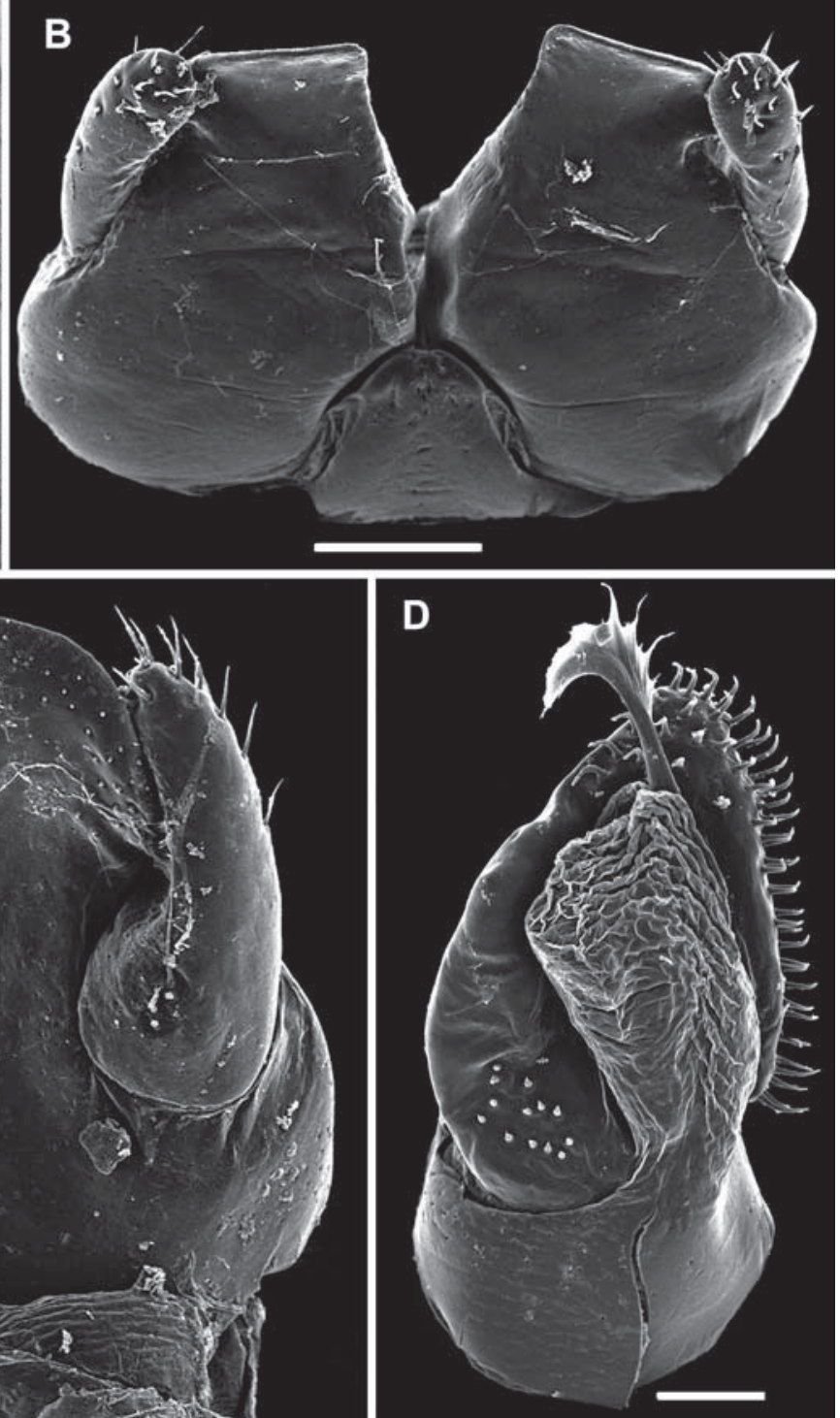

Fig. 9. Glyphiulus parechinoides sp.n., O paratype: A - enlarged ozopore region, lateral view; B \& C - anterior gonopods, anteroventral and caudal views, respectively; D - left posterior gonopod, front view. Scale bars: A-C - $0.1 \mathrm{~mm}$; D - $0.05 \mathrm{~mm}$.

Рис. 9. Glyphiulus parechinoides sp.n., паратип $\sigma^{7}$ : А - увеличенный район озопоры; В, С - передние гоноподы, соотвественно спереди и снизу, а также сзади; D - левый задний гонопод, спереди. Масштаб: А-C — 0,1 мм; D - 0,05 мм.

Postcollum constriction rather evident. All crests on collum complete and evident; carinotaxy formula, I-III+P+M (Figs 1A, D).

Subsequent metaterga strongly crested (Figs 1, 2F); carinotaxy formula, $2 / 2+\mathrm{I} / \mathrm{i}+3 / 3$. Ozoporiferous cones especially large, about as high as broad, directed caudolaterad (like all others), rather narrowly truncate on top; midbody crests divided into two about midway, both halves being sharp and nearly as high as ozoporiferous cones.

Tegument finely alveolate-areolate, dull throughout (Fig. 2G). Fine longitudinal striations in front of stricture between pro- and metazona, remaining surface of prozona very delicately shagreened (Figs 1B, D). Segments 2 and 3 with long pleural flaps (Fig. 1A).
Midbody segments slightly compressed laterally (Fig. 2F). Limbus extremely finely and regularly denticulate/ spiculate. Epiproct (Figs 1C, F, 2E) simple, regularly rounded caudally, with a strong central tooth dorsally. Paraprocts rather regularly convex (Figs 2D, E), median margins evidently elevated due to premarginal sulci. Hypoproct transversely lanceolate, paramedian setigerous pits (setae abraded) nearly contiguous (Fig. 2D).

Ventral flaps behind gonopod opening on segment 7 rather poorly distinguishable as low swellings, not forming a marked transverse ridge.

Legs rather short (Figs 2F), on midbody segments about 3/4 length of segment height. Claw at base with a strong accessory spine more than half as long as claw itself (Fig. 2H). 


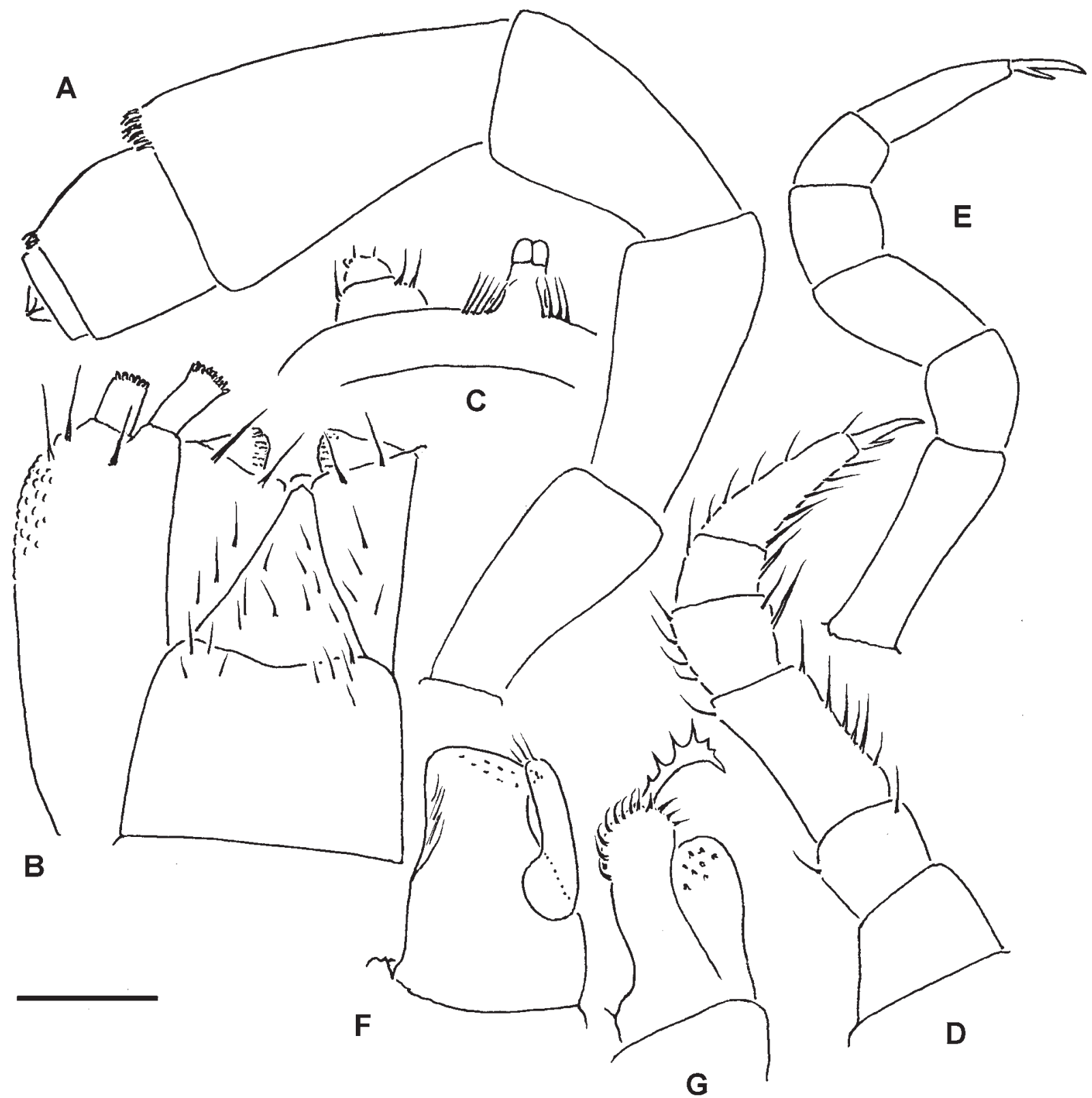

Fig. 10. Glyphiulus parechinoides sp.n., O paratype: A - antenna, lateral view; B - gnathochilarium, ventral view; C - legs 1, front view; D - leg 2, caudal view; E — leg 3, caudal view; F — right anterior gonopod, caudal view; G — right posterior gonopod, caudal view. Scale bars: $0.2 \mathrm{~mm}$.

Рис. 10. Glyphiulus parechinoides sp.n., паратип О’: A - антенна, сбоку; В — гнатохилярий, снизу; C - ноги 1, спереди; D нога 2, сзади; Е - нога 3, сзади; F — левый передний гонопод, сзади; G — правый задний гонопод, сзади. Масштаб: 0,2 мм.

$\sigma^{7}$ legs 1 (Fig. 4C) represented by a sternum showing a pair of small, fused, paramedian, subunciform prongs directed forward, flanked by strongly separated, rudimentary, 2-segmented leg vestiges. $\sigma^{\gamma}$ legs 2 only barely hypertrophied (Fig. 4D); penes small, oblong-subtrapeziform, each with two strong setae distolaterally. $\sigma^{\gamma}$ legs 3 modified in having coxa especially slender and elongate. $\sigma^{7}$ femora 6 or 7 not inflated ventrally.

Anterior gonopods (Figs 3A, B, 4E) with a typical shield-like coxosternum, this being rather sparsely mi- crosetose on caudal face, lower than 1-segmented telopodites. Posterior gonopods (Figs 3C, D, 4F) as usual, very compact, coxite medio-apically with a plumose process (= flagellum).

\section{Glyphiulus subechinoides sp.n.}

Figs 5-7.

MATERIAL. Holotype $\sigma^{7}$ (NMNHS), Vietnam, Quang Ninh Prov., Ha Long Bay, Bo Hon Island, $15 \mathrm{~km} \mathrm{~S}$ of Bai Chay Beach, Cave Hong Luôn, hand collection, 1.03.1989, leg. P. Beron \& D. Kojuharov. Paratypes: $3 \sigma^{7} \sigma^{7}, 5$ juv. (NMNHS), $1 \sigma^{7}, 1$ (MNHN 


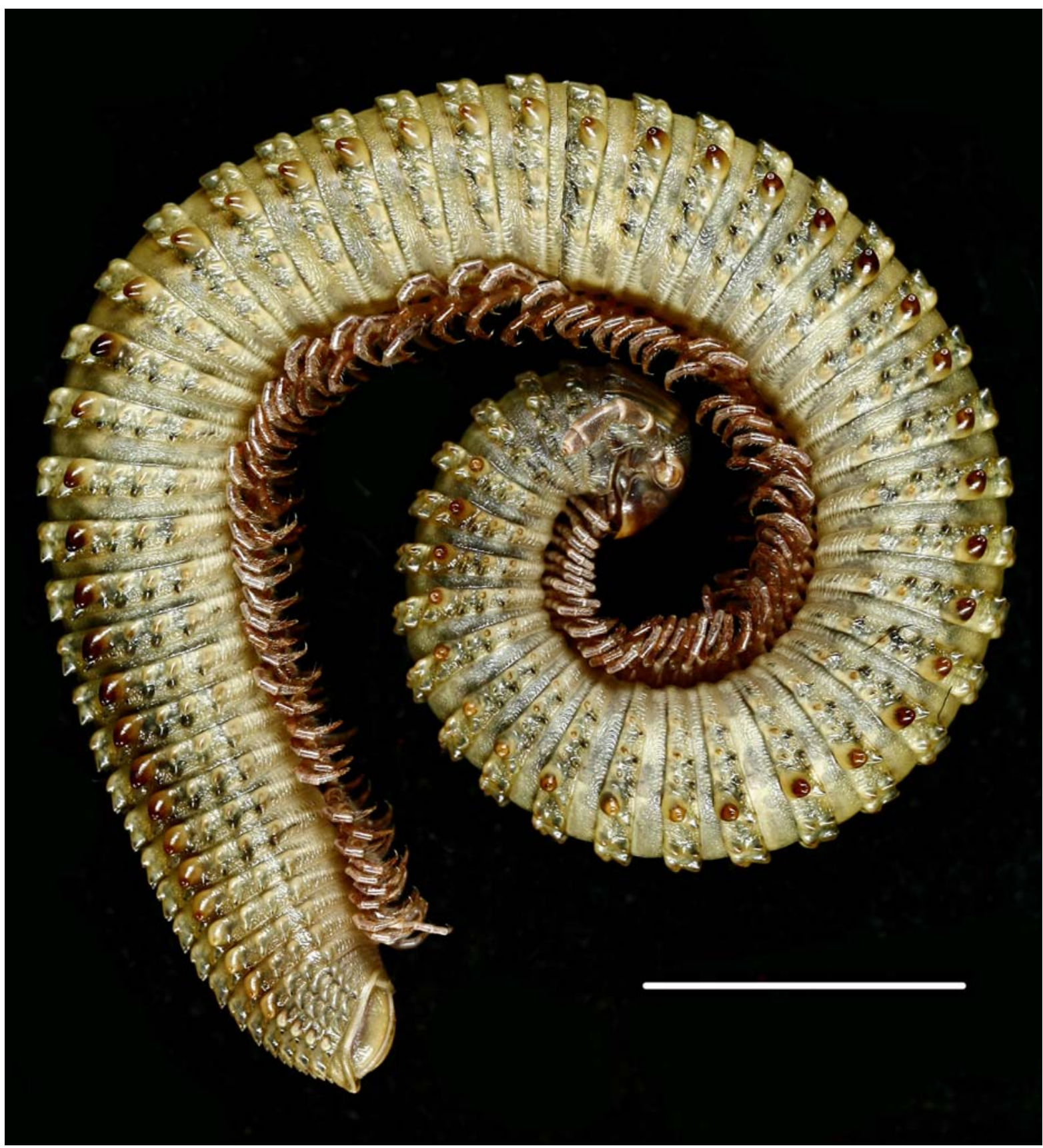

Fig. 11. Glyphiulus submediator sp.n., + paratype. Habitus, lateral view. Scale bar: $5.0 \mathrm{~mm}$.

Рис. 11. Glyphiulus submediator sp.n., паратип +. Габитус, сбоку. Масштаб: 5,0 мм.

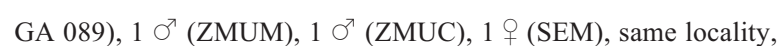
together with holotype.

NAME. To emphasize the similarity to G. echinoides sp.n.

DIAGNOSIS. Comes closest to the previous species, but differs in the longer antennae and legs, the inflated $\sigma^{7}$ femora 6 and 7, the higher coxosternum of the anterior gonopods, etc. Shares the inflated $\sigma^{7}$ femora 6 and 7 with $G$. recticullus Zhang \& Li, 1982, from Zhejiang Province, China [Zhang \& Li, 1982], but distinguished by the body slightly compressed lateral- ly, in the crests/tubercles on metazona being sharper and higher, as well as by the shape of the anterior gonopod coxosternum.

DESCRIPTION. Length ca 30-33 mm, width 1.3$1.5 \mathrm{~mm}$, with $52-59 \mathrm{p}+3-1 \mathrm{a}+\mathrm{T}$, midbody segments being broadest. Holotype ca $32 \mathrm{~mm}$ long, $1.3 \mathrm{~mm}$ wide, with $58 \mathrm{p}+1 \mathrm{a}+\mathrm{T}$. Coloration uniformly yellow to light yellow-brown.

All characters as in G. echinoides sp.n. (Figs 5-7), except as follows. Light brown ocelli arranged in two vertical rows of 5+2(3) (Fig. 5A). Antennae long and 

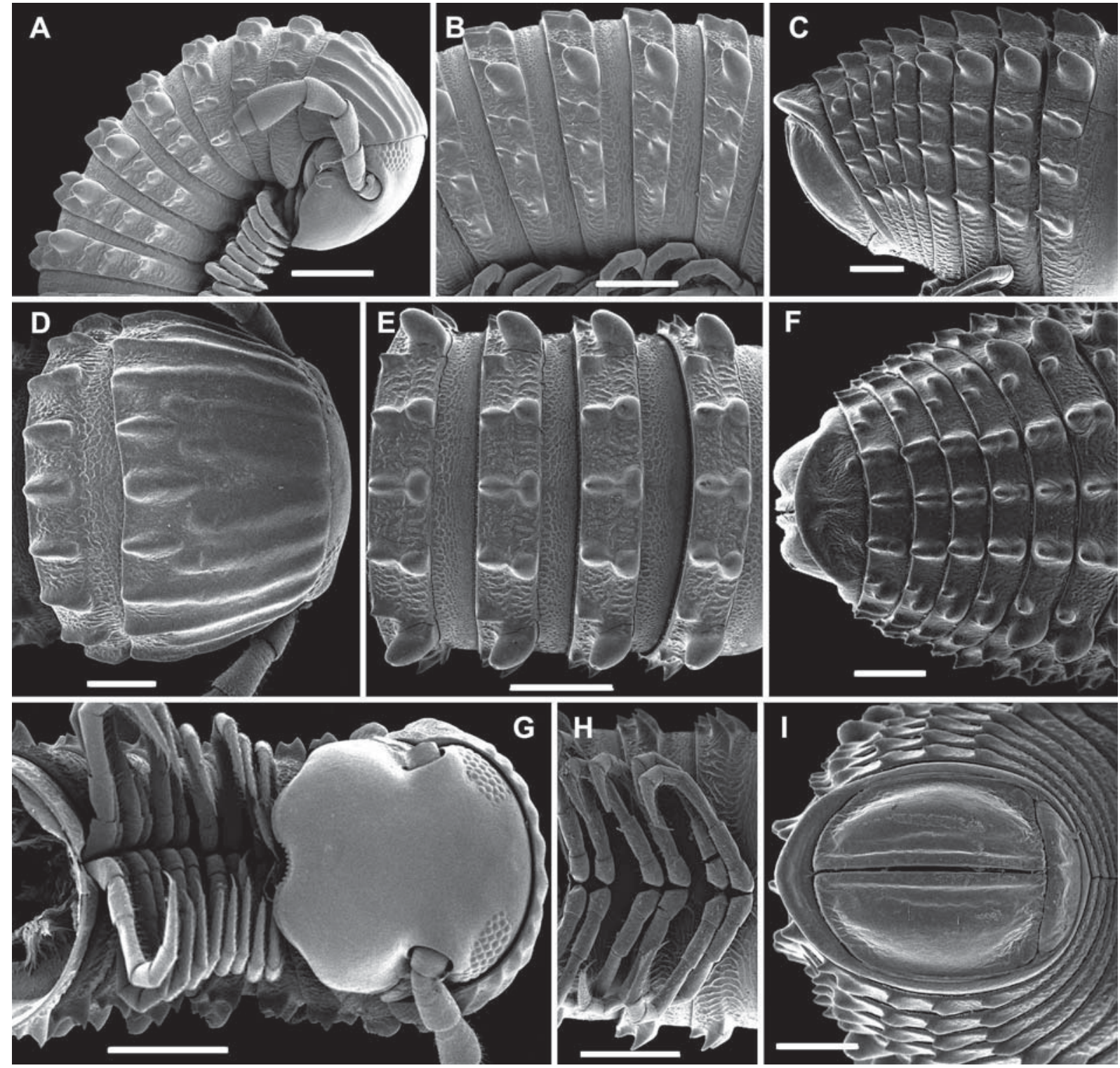

Fig. 12. Glyphiulus submediator sp.n., , paratype: A, D \& G — anterior part of body, lateral, dorsal and ventral views, respectively; B, E \& H - midbody segments, lateral, dorsal and ventral views, respectively; C, F \& I - posterior part of body, lateral, dorsal and ventral views, respectively. Scale bars: $0.5 \mathrm{~mm}$.

Рис. 12. Glyphiulus submediator sp.n., паратип 9 : A, D, G - передняя часть тела, соответственно сбоку, сверху и снизу; В, Е, H - среднетуловищные сегменты, соответственно сбоку, сверху и снизу; C, F, I - задняя часть тела, соответственно сбоку, сверху и снизу. Масштаб: 0,5 мм.

moderately clavate (Figs 5A, B, 7A), extending beyond segment 4 laterally. Gnathochilarium with a separate promentum, oligotrichous (Figs 6A, 7B).

Legs rather long and slender (Figs 5G, 6B, E), on midbody segments about 1.2-1.3 length of segment height. Claw at base with a strong accessory spine more than half as long as claw itself (Fig. 6F).

$\sigma^{7}$ legs 1 (Fig. 7C) represented by a sternum showing a pair of small, fused, paramedian, subunciform prongs directed forward, flanked by strongly separated, rudimentary, 2-segmented leg vestiges. $\sigma^{7}$ legs 2 only barely hypertrophied (Fig. 7D); penes small, oblong-subtrapeziform, each with three strong setae distolaterally. $\sigma^{7}$ legs 3 modified in having coxa especially slender and elongate (Fig. 7E). $\sigma^{7}$ femora 6 and 7 ventrally inflated and densely pubescent in distal half (Fig. 7F).

Anterior gonopods (Fig. 7G) with a typical shieldlike coxosternum, this being rather densely microsetose on caudal face, higher than 1-segmented telopodites. Posterior gonopods (Fig. $7 \mathrm{H}$ ) very compact, coxite medio-apically with a plumose flagellum.

\section{Glyphiulus parechinoides sp.n. \\ Figs 8-10.}

MATERIAL. Holotype $O^{\top}$ (NMNHS), Vietnam, Langson Prov., Hong Phong Village, Cave Mûoi, hand collection, 13.03.1989, leg. P. Beron \& D. Kojuharov. Paratypes: complete $1 \sigma^{7}, 4$ of , numerous fragmented $\sigma^{7}, q \&$ juv. (NMNHS), fragmented $2 \sigma^{7} \sigma^{7}, 2$ 우 (MNHN 

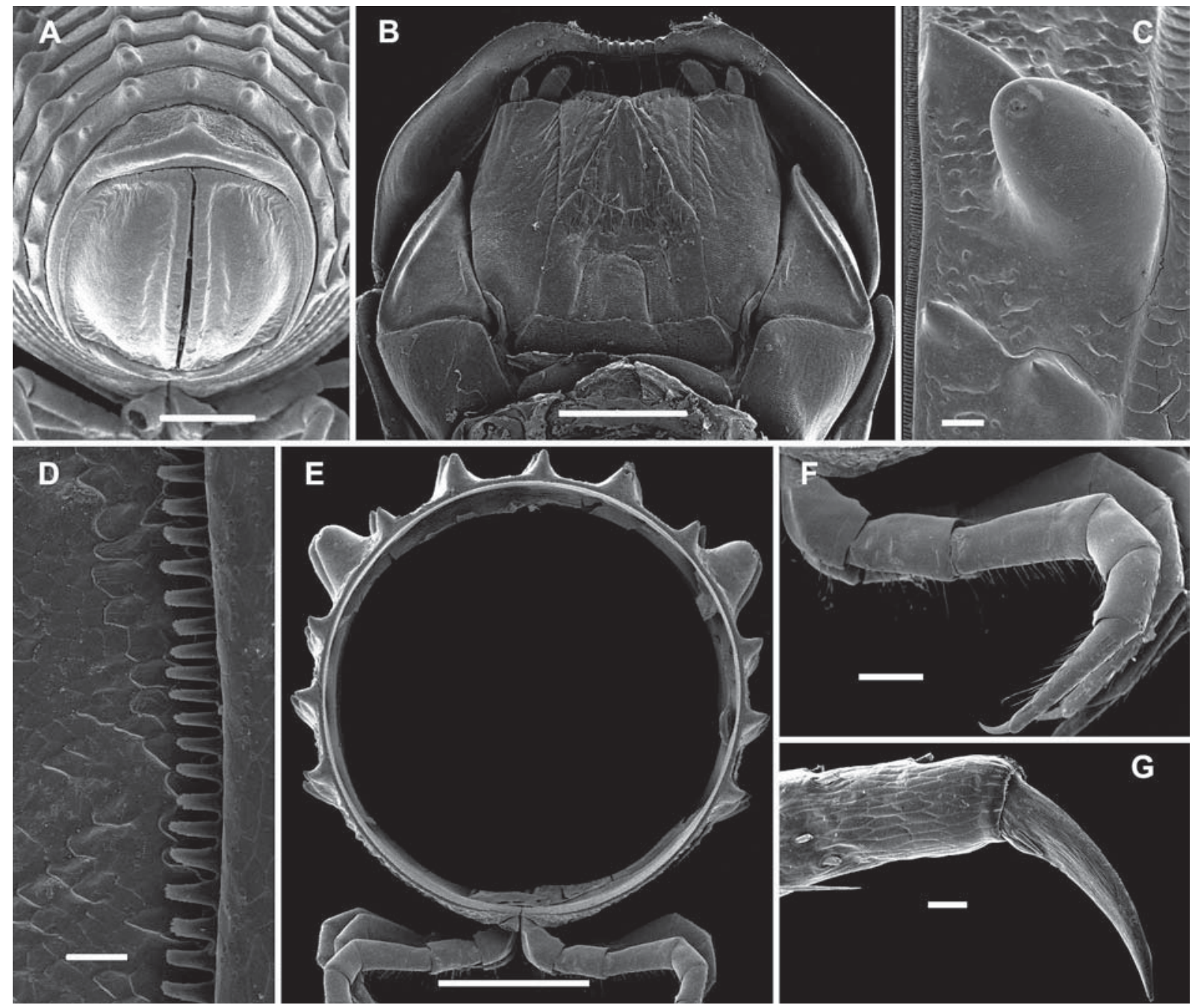

Fig. 13. Glyphiulus submediator sp.n.,, paratype: A — posterior part of body, caudal view; B — gnathochilarium, ventral view; C enlarged ozopore region, lateral view; D - limbus, lateral view; E — cross-section of a midbody segment, caudal view; F — midbody legs, caudal view; $\mathrm{G}$ - enlarged claw, lateral view. Scale bars: A, B, E - $0.5 \mathrm{~mm} ; \mathrm{C}, \mathrm{F}-0.1 \mathrm{~mm}$; D, G - $0.02 \mathrm{~mm}$.

Рис. 13. Glyphiulus submediator sp.n., паратип +: А - задняя часть тела, сзади; В - гнатохилярий, снизу; С - увеличенный район озопоры, сбоку; D - лимбус, сбоку; Е — поперечный разрез через среднетуловищный сегмент, сзади; F — среднетуловищные ноги, сзади; G - увеличенный коготок, сбоку. Масштаб: А, B, E-0,5 мм; C, F-0,1 мм; D, G-0,02 мм.

GA 090), fragmented $2 \bigcirc^{\top} \sigma^{7}, 2$ 우 (ZMUM), fragmented $1 \odot^{\top}, 19$ (ZMUC), $1 O^{\top}$ (SEM), same locality, together with holotype.

NAME. To emphasize the similarity to $G$. echinoides sp.n. and G. subechinoides sp.n.

DIAGNOSIS. Especially similar to $G$. echinoides sp.n., but differs in the polytrichous gnathochilarium, the round cross-section of midbody segments, the slightly longer antennae and legs, the lower tubercles/crests on metazona, the subequally high coxosternum and telopodites of the anterior gonopods, etc. Shares with $G$. recticullus and $G$. subechinoides sp.n. the inflated $\sigma^{7}$ femora 6 and 7 , but distinguished from the former by the considerably lower, sharper and narrower metazonital crests/tubercles, from the latter by the midbody segments not being compressed laterally.

DESCRIPTION. Length of complete specimens ca 39-43 mm, width $1.7-1.9 \mathrm{~mm}$ (some fragments up to
$2.0 \mathrm{~mm}$ wide), with $60-70 \mathrm{p}+2-1 \mathrm{a}+\mathrm{T}$, midbody segments being broadest. Holotype complete, ca $42 \mathrm{~mm}$ long, $1.9 \mathrm{~mm}$ wide, with $68 \mathrm{p}+2 \mathrm{a}+\mathrm{T}$. Coloration usually uniformly light yellow-brown to dark brown, ozoporiferous cones sometimes infuscate, up to black-brown.

All characters as in G. echinoides sp.n. (Figs 8-10), except as follows. Brown to black-brown ocelli arranged in two vertical row of 7(8)+5(6) (Fig. 8A). Antennae rather long and moderately clavate (Figs 8A, 10A), extending beyond segment 4 laterally. Gnathochilarium with a separate promentum, polytrichous (Figs 8J, 10B).

Crests/tubercles on metazona sharp, narrow, rather low (Figs 8A-F, H, I, K).

Legs rather long and slender (Fig. 8L), on midbody segments about 1.1-1.2 length of segment height. Claw at base with a strong accessory spine more than half as long as claw itself (Fig. 8M). 


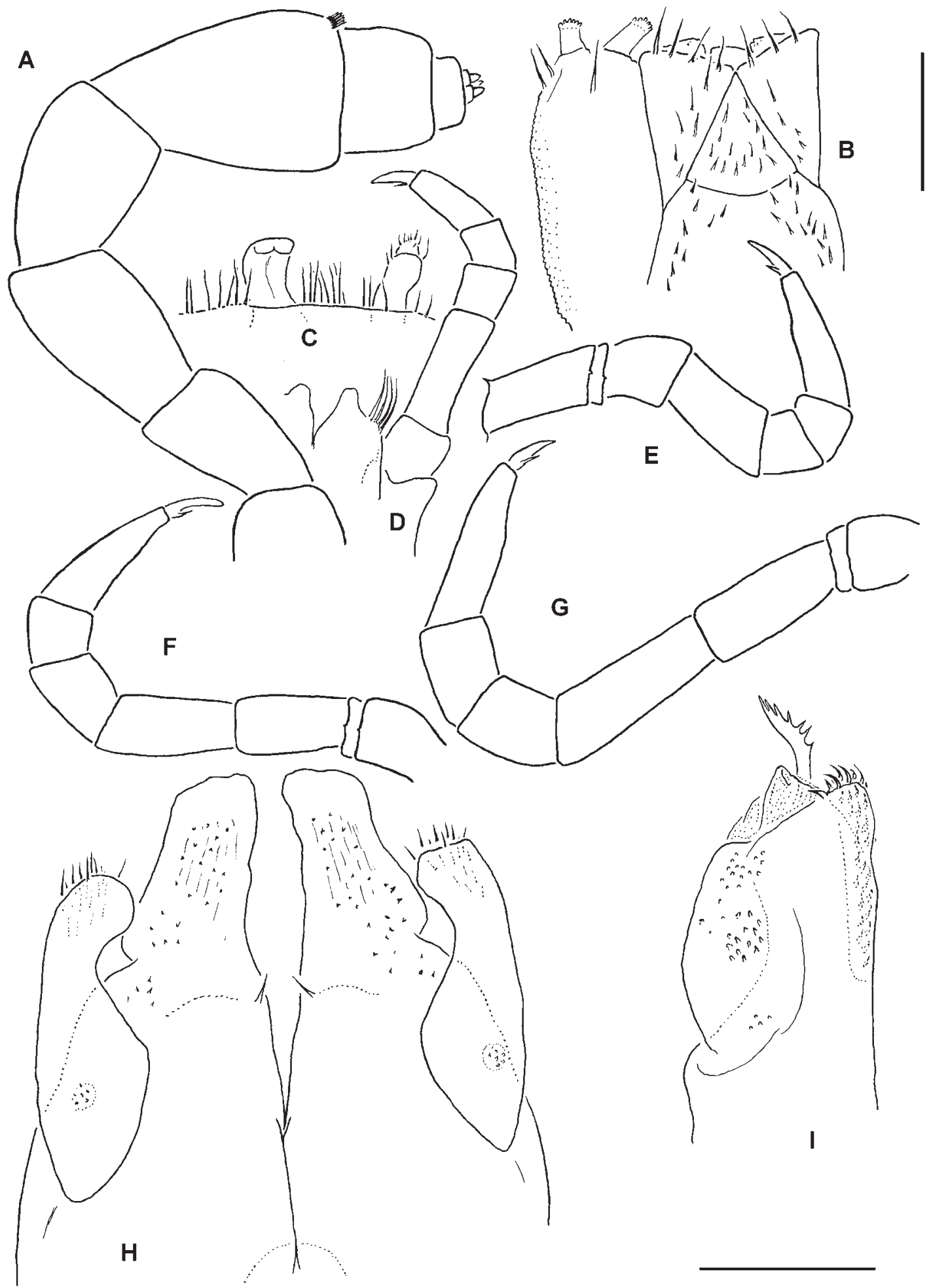

Fig. 14. Glyphiulus submediator sp.n., O holotype: A — antenna, lateral view; B - gnathochilarium, ventral view; C - legs 1, front view; D — leg 2, caudal view; E — leg 3, caudal view; F \& G — midbody legs, lateral views; H — anterior gonopods, caudal view; I — left posterior gonopod, caudal view. Scale bars: $\mathrm{A}-\mathrm{G}-0.4 \mathrm{~mm} ; \mathrm{H}, \mathrm{I}-0.2 \mathrm{~mm}$.

Рис. 14. Glyphiulus submediator sp.n., голотип Оج: А — антенна, сбоку; В — гнатохилярий, снизу; С — ноги 1, спереди; D нога 2, сзади; Е - нога 3, сзади; F, G - среднетуловищные ноги, сбоку; H - передние гоноподы, сзади; I - левый задний гонопод, сзади. Масштаб: A-G - 0,4 мм; H, I-0,2 мм. 

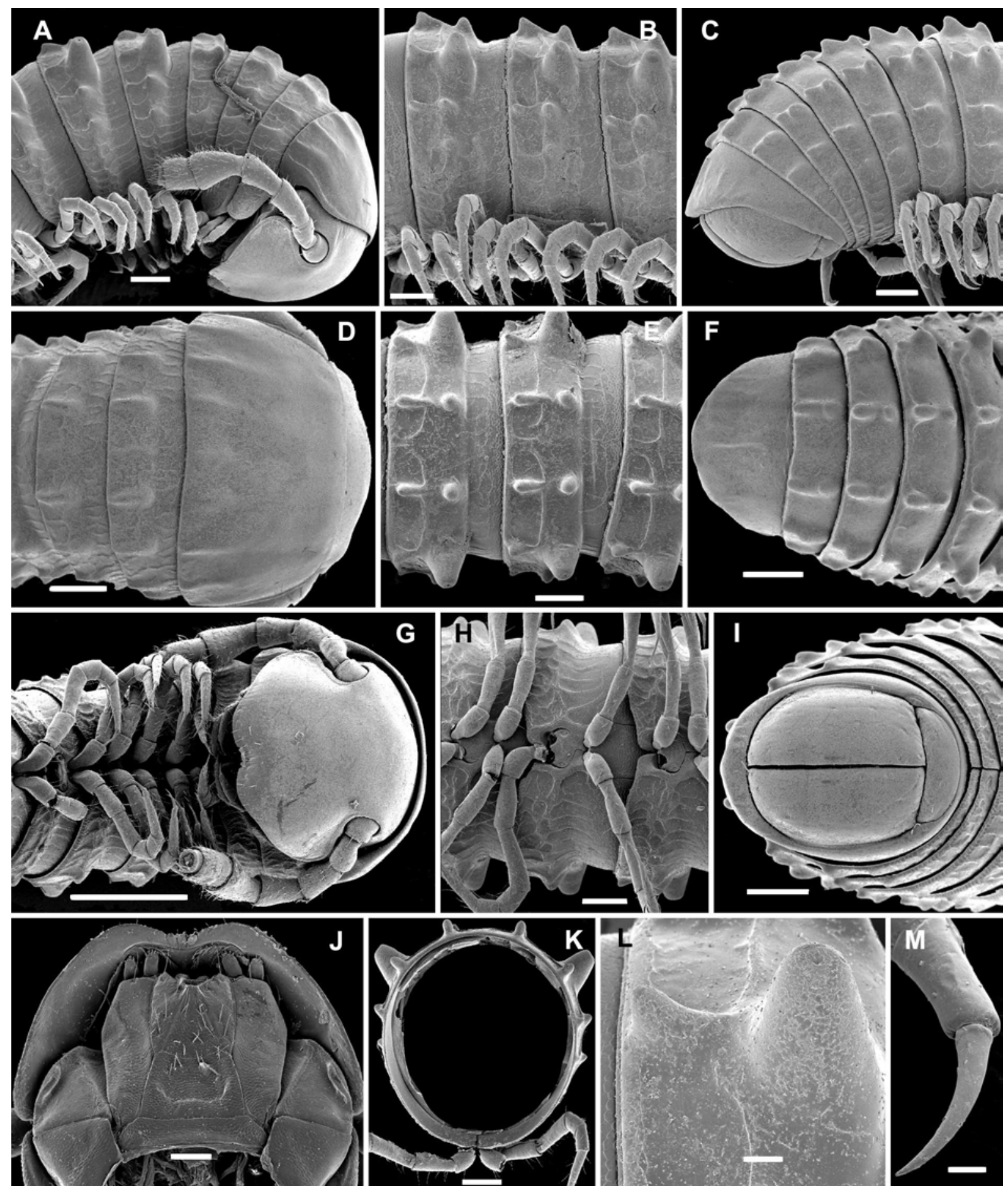

Fig. 15. Glyphiulus mediobliteratus Golovatch, Geoffroy, Mauriès \& VandenSpiegel, 2007, 9 from Cave Hang Nuoc: A, D \& G anterior part of body, lateral, dorsal and ventral views, respectively; B, E \& $\mathrm{H}$ - midbody segments, lateral, dorsal and ventral views, respectively; C, F \& I - posterior part of body, lateral, dorsal and ventral views, respectively; J - gnathochilarium, ventral view; K cross-section of a midbody segment, caudal view; L — enlarged ozopore region, lateral view; $\mathrm{M}$ — enlarged claw, lateral view. Scale bars: A-F, H, I, K $-0.2 \mathrm{~mm}$; G $-0.5 \mathrm{~mm}$; J $-0.1 \mathrm{~mm} ; \mathrm{L}-0.05 \mathrm{~mm} ; \mathrm{M}-0.02 \mathrm{~mm}$

Рис. 15. Glyphiulus mediobliteratus Golovatch, Geoffroy, Mauriès \& VandenSpiegel, 2007, + из пещеры Hang Nuoc: A, D, G передняя часть тела, соответственно сбоку, сверху и снизу; В, Е, Н - среднетуловищные сегменты, соответственно сбоку, сверху и снизу; С, F, I - задняя часть тела, соответственно сбоку, сверху и снизу; J — гнатохилярий, снизу; К — поперечный разрез через среднетуловищный сегмент, сзади; L — увеличенный район озопоры, сбоку; $\mathrm{M}$ - увеличенный коготок, сбоку. Масштаб: A-F, H, I, K-0,2 мм; G-0,5 мм; J-0,1 мм; L - 0,05 мм; M - 0,02 мм. 


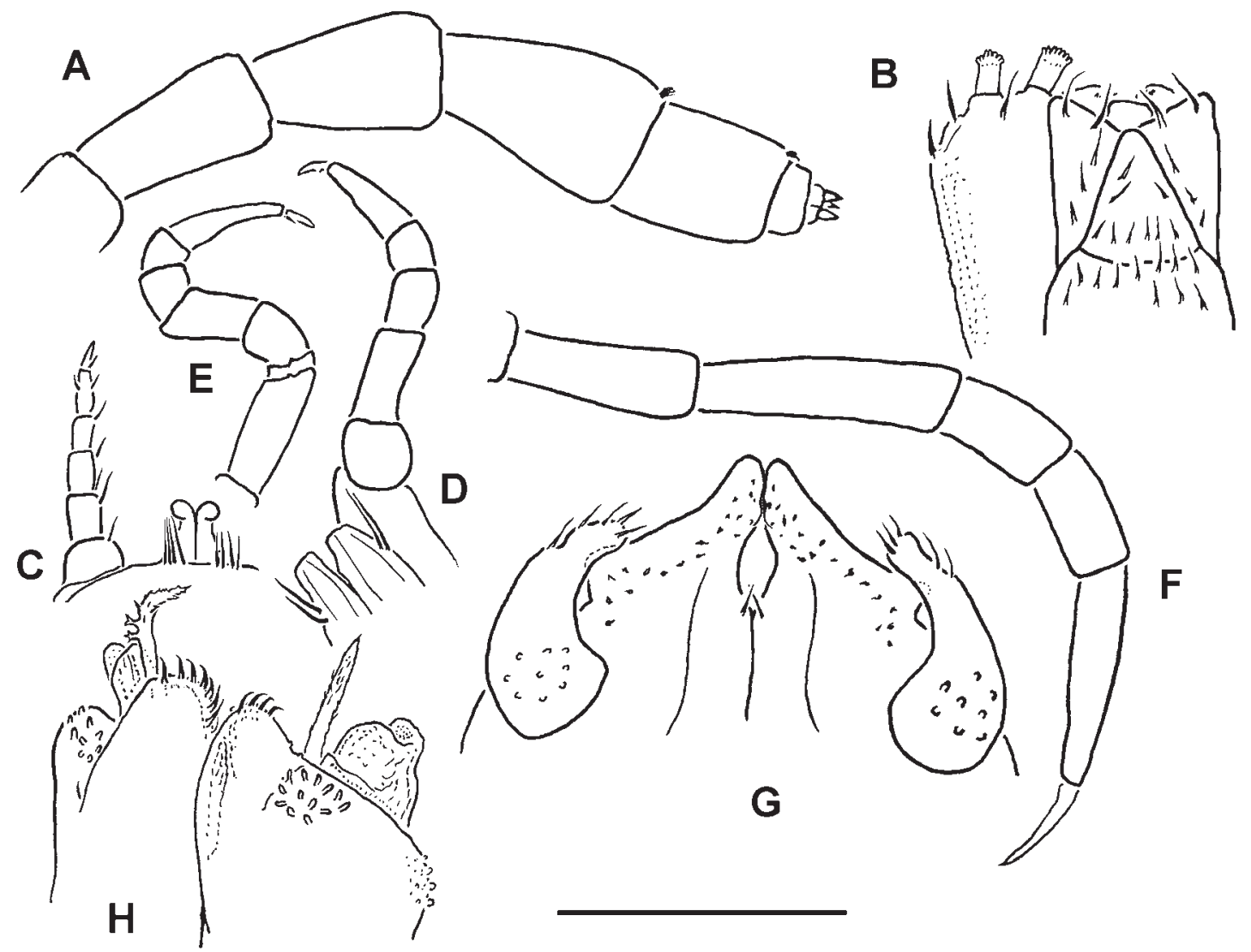

Fig. 16. Glyphiulus mediobliteratus Golovatch, Geoffroy, Mauriès \& VandenSpiegel, 2007, $0^{7}$ from Cave Hang Nuoc: A — antenna, lateral view; B - gnathochilarium, ventral view; C - legs 1, front view; D - leg 2, caudal view; E - leg 3, caudal view; F - midbody leg, lateral view; $\mathrm{G}$ - anterior gonopods, caudal view; $\mathrm{H}$ - posterior gonopods, caudolateral view. Scale bars: A-F $-0.4 \mathrm{~mm} ; \mathrm{G}, \mathrm{H}-$ $0.2 \mathrm{~mm}$.

Pис. 16. Glyphiulus mediobliteratus Golovatch, Geoffroy, Mauriès \& VandenSpiegel, 2007, О из пещеры Hang Nuoc: A антенна, сбоку; В — гнатохилярий, снизу; С - ноги 1, спереди; D - нога 2, сзади; Е - нога 3, сзади; F — среднетуловищная нога, сбоку; G - передние гоноподы, сзади; H — задние гоноподы, сзади и сбоку. Масштаб: A-F - 0,4 мм; G, H - 0,2 мм.

$\sigma^{7}$ legs 1 (Figs 8J, 10C) represented by a sternum showing a pair of small, fused, paramedian, subunciform prongs directed forward, flanked by strongly separated, rudimentary, 2-segmented leg vestiges. $\sigma^{7}$ legs 2 only barely hypertrophied (Fig. 10D); penes small, oblongsubtrapeziform, each with three strong setae distolaterally. $\sigma^{7}$ legs 3 modified in having coxa especially slender and elongate (Fig. 10E). $\sigma^{7}$ femora 6 and 7 more like in G. recticullus, bulged ventrally in distal third, bare.

Anterior gonopods (Figs 9B, C, 10F) with a typical shield-like coxosternum, this being rather densely microsetose on caudal face, about as high as 1-segmented telopodites. Posterior gonopods (Figs 9D, 10G) very compact, coxite medio-apically with a plumose flagellum.

\section{Glyphiulus submediator sp.n.}

Figs 11-14.

MATERIAL. Holotype $\sigma^{\top}$ (MNHN GA 091), Vietnam, Lam Dong Prov., Dalat, Bidoup National Park, Hon Giao, forest litter,
11.06.2008, leg. L. Deharveng \& A. Bedos (Vn08-112). Paratype: 1 ( (SEM), same locality, together with holotype.

NAME. To emphasize the similarity to G. mediator Attems, 1938.

DIAGNOSIS. Comes closest to $G$. mediator, from Bana, central Vietnam [Attems, 1938], based on size, coloration, gonopod structure etc., but differs in the peculiar carinotaxy formulas of the collum and metazona, the less stout antennomere 5 etc.

DESCRIPTION. Length of holotype ca $46 \mathrm{~mm}$, width $2.6 \mathrm{~mm}$, with $58 \mathrm{p}+1 \mathrm{a}+\mathrm{T}$, midbody segments being broadest. Coloration of holotype variegated marbled dark black-brown with reddish blotches; legs redbrown; coloration of paratype uniformly dark greybrown (Fig. 11).

Body strongly robust (Fig. 11). Ocellaria transversely ovoid, each composed of ca 25 blackish ocelli arranged in seven vertical rows (Fig. 12G). Antennae short and moderately clavate (Figs 12A, 14A), extending beyond segment 3 laterally, antennomere 5 not 
markedly higher than $4^{\text {th }}$, with a small distodorsal field of bacilliform sensilla. Gnathochilarium with a separate promentum, polytrichous (Figs 13B, 14B).

Postcollum constriction rather evident. All crests on collum complete, but evident only in caudal halves, mid-dorsal ones (especially paramedians) somewhat obliterated anteriorly; carinotaxy formula, I-III $+4 \mathrm{c}+$ $5 \mathrm{a}+\mathrm{pc}+$ ma (Figs 12A, D).

Subsequent metaterga strongly crested (Figs 12AF, 13E); carinotaxy formula, $3 / 3+\mathrm{I} / \mathrm{i}+3 / 3$. Ozoporiferous cones especially large, about as high as broad, directed caudolaterad (like all others), usually rather regularly rounded on top; midbody crests divided into two about midway, both halves mostly being narrowly rounded and lower than ozoporiferous cones.

Midbody segments round in cross-section (Fig. 13E). Limbus finely and regularly denticulate (Fig. 13D). Epiproct (Figs 12C, F, 13A) simple, regularly rounded caudally, without evident structures dorsally. Paraprocts rather regularly convex (Figs 12C, 13A), median margins evidently elevated due to premarginal sulci. Hypoproct transversely lanceolate (Fig. 12I).

Legs rather short (Figs 13E, F, 14F, G), on midbody segments about length of segment height. Claw at base with a short accessory spine (Fig. 13G).

$\sigma^{7}$ legs 1 (Fig. 14C) represented by a sternum showing a pair of small, fused, paramedian, subunciform prongs directed forward, flanked by strongly separated, rudimentary, 2-segmented leg vestiges. $\sigma^{7}$ legs 2 only barely hypertrophied (Fig. 14D); penes small, bottle-shaped, each with four strong setae distolaterally. $\sigma^{7}$ legs 3 modified in having coxa rather slender and elongate (Fig. 14E). $\sigma^{7}$ femora 6 or 7 not inflated ventrally.

Anterior gonopods (Fig. 14H) with a typical shieldlike coxosternum, this being rather densely microsetose on caudal face, high, subquadrate, much higher than 1-segmented telopodites. Posterior gonopods (Fig. 14I) as usual, very compact, coxite medio-apically with a plumose flagellum.

Glyphiulus mediobliteratus Golovatch, Geoffroy, Mauriès \& VandenSpiegel, 2007 Figs 15-16.

MATERIAL. $30^{7} \sigma^{7}, 1$ (MNHN GA 046), $1 \bigcirc^{7}$ (ZMUC), 19 (SMF), Vietnam, Thanh Hoa Prov., Thanh Yen Distr., Cave Hang Nuoc near Cuc Phuong, hand collection, 8.12.2003, leg. L. Deharveng et al. (Vn0312-02).

REMARKS. The above new samples agree very well with the types, which are also known from caves in the Cuc Phuong region, northeastern Vietnam [Golovatch et al., 2007b]. To demonstrate conspecificity, abundant illustrations are provided (Figs 15-16). Slight variations concern the new material containing smaller specimens (length 19-25 mm, width 1.1-1.5 mm, with $39-44 p+3-2 a+T)$, mostly pallid to light yellow in coloration (only tips of some ozoporiferous cones sometimes infuscate, brown to dark brown), somewhat longer $\sigma^{7}$ telopodites 1 etc.

ACKNOWLEDGEMENTS. This work only became possible through the support offered to the first author by the Muséum national d'Histoire naturelle, Paris. Both Louis Deharveng and Anne Bedos (both MNHN), as well as Petar Beron (NMNHS) are deeply thanked for the precious material they provided for study and for donating it entirely or in part to the MNHN. Nguyen Duc Anh (Hanoi, Vietnam) kindly helped in providing additional information concerning one of the localities.

\section{References}

Attems C. 1938. Die von Dr C. Dawydoff in Französisch Indochina gesammelten Myriopoden // Mémoires du Muséum national d'Histoire naturelle. N.S. T.6. Fasc.2. P.187-353.

Golovatch S.I., Geoffroy J.-J., Mauriès J.-P., VandenSpiegel D. 2007a. Review of the millipede genus Glyphiulus Gervais, 1847, with descriptions of new species from Southeast Asia (Diplopoda, Spirostreptida, Cambalopsidae). Part 1. The granulatus-group // Zoosystema. Vol.29. Fasc.1. P.7-49.

Golovatch S.I., Geoffroy J.-J., Mauriès J.-P., VandenSpiegel D. 2007b. Review of the millipede genus Glyphiulus Gervais, 1847, with descriptions of new species from Southeast Asia (Diplopoda, Spirostreptida, Cambalopsidae). Part 2. The javanicus-group // Zoosystema. Vol.29. Fasc.3. P.417-456.

Golovatch S.I., Geoffroy J.-J., Mauriès J.-P., VandenSpiegel D. 2011a. The millipede genus Plusioglyphiulus Silvestri, 1923 in Thailand (Diplopoda, Spirostreptida, Cambalopsidae) // Zootaxa. No.2940. P.1-63.

Golovatch S.I., Geoffroy J.-J., Mauriès J.-P., VandenSpiegel D. 2011 b. New species of the millipede genus Glyphiulus Gervais, 1847 from the granulatus-group (Diplopoda: Spirostreptida: Cambalopsidae) // Arthropoda Selecta. Vol.20. No.2. P.65-114.

Golovatch S.I., Geoffroy J.-J., Mauriès J.-P., VandenSpiegel D. 2011c. Two new species of the millipede genus Hypocambala Silvestri, 1895 from China and Vietnam (Diplopoda: Spirostreptida: Cambalopsidae) // Arthropoda Selecta. Vol.20. No.3. P.167-174.

Jeekel C.A.W. 1963. Diplopoda of Guiana (1?5) // Studies on the Fauna of Suriname and other Guyanas. Vol.4. No.11. P.1-157.

Jeekel C.A.W. 2004. A bibliographic catalogue of the "Cambaloidea“ (Diplopoda, Spirostreptida) // Myriapod Memoranda. Vol.7. P.43-109.

Loksa I. 1960. Einige neue Diplopoden- und Chilopodenarten aus chinesischen Höhlen // Acta Zool. Acad. Sci. Hung. T.6. Fasc.12. P.137-148.

Mauriès J.-P. 1977. Le genre Glyphiulus Gervais, 1847, et sa place dans la classification des Cambalides, à propos de la description d'une nouvelle espèce du Viêt-Nam (Diplopoda, Iulida, Cambalidea) // Bulletin du Muséum national d'Histoire naturelle. 3e sér. No.431 (Zool. 301). P.243-250.

Zhang C.Z., Li Z.Y. 1982. Die Gattung Glyphiulus (Diplopoda: Cambalidea) von China // Sinozoologia. Vol.2. P.85-93 [in Chinese, with German summary].

Responsible editor K.G. Mikhailov 